\title{
How Stakeholders' Commitment May Affect the Success of Requirements Elicitation
}

\author{
Corentin Burnay* ${ }^{* \dagger}$, Ivan Jureta* ${ }^{* \dagger}$ and Stéphane Faulkner ${ }^{\dagger \ddagger}$ \\ ${ }^{*}$ Fonds de la Recherche Scientifique - FNRS, Brussels \\ $\dagger$ Department of Business Administration, University of Namur \\ ${ }_{\ddagger}^{\ddagger}$ PReCISE Research Center, University of Namur
}

\begin{abstract}
Requirements elicitation consists in collecting information about the requirements and the environment of a systemto-be. It usually involves business analysts who are eliciting information, and stakeholders who are providing information. This paper investigates how the commitment of stakeholders to a RE project influences the results of elicitation. We suggest a way to measure the commitment of stakeholders during RE, and propose the so-called "commitment matrix", which shows what analysts can expect from stakeholders who are more, as opposed to those who are less committed. The matrix builds on a survey of 87 stakeholders. Our results suggest that commitment somehow affects the information provided by stakeholders, and that it is therefore a relevant criterion to account for when selecting stakeholders to be involved in elicitation.
\end{abstract}

Keywords-Requirements Elicitation, Commitment, Involvement

\section{INTRODUCTION}

1) Context: Requirements Engineering (RE) focuses on the elicitation, representation, and analysis of requirements and environment of a system-to-be, in order to produce its specification. The specification should be such that, if the systemto-be satisfies the specification, then it will also satisfy its requirements within its environment. RE involves various tasks, such as the elicitation of requirements, their representation, the analysis of their consistency, their validation and negotiation with the system stakeholders, and so on. We focus in this paper on the elicitation aspect; that is, on the acquisition of information about the expectations of stakeholders toward the system-to-be, and about the environment in which that system will run. We will say that this information is represented in elicitation documentation, such as interview transcripts or recordings, notes from field observation, questionnaires and similar. There are at least three recurring challenges that should be considered when preparing the elicitation documentation:

- Documentation Quality: information must be easily understandable, and should be sufficiently stable so that analysts can actually rely on the information [1], [2];

- Documentation Quantity: information must cover the relevant requirements of the stakeholders, and should not overlook important aspect of the environment, so as to minimize requirements incompleteness [1], [2], [3];

- Documentation Efficiency: information must be obtained at reasonable cost and resource use, i.e., it is important to achieve quality and quantity, but not at any cost [4].

(DOI reference number: 10.18293/SEKE2015-105)
In the rest of this paper, we refer to these three Requirements Elicitation Challenges as RECs. How successfully one deals with REC is influenced, among other things, by the choice of some elicitation techniques to apply [5] and the selection of stakeholders to apply them with [6], [7].

2) Problem and Research Question: Identifying stakeholders is hardly a new issue in RE [8]; most of the time, a person is considered as a stakeholder whenever she has a stake in the system [9]. In RE, it has been suggested that a stakeholder is any person or organization who influences a system's requirements or who is impacted by that system [10]. Such definition likely leads to a large set of stakeholders, which can hardly be entirely solicited. The question of selecting stakeholders to be involved from the pool of available stakeholders is, to the best of our knowledge, a topic that has received smaller attention from RE community. More precisely, we believe that one possible way of selecting some stakeholders is to account for their respective level of commitment to the RE project, i.e., how the stakeholders are intellectually or emotionally bounded to the RE project. For instance, involving two stakeholders with different commitment levels during elicitation could result in elicitation documents that differ in terms of quality, quantity or efficiency. In fact, the committed stakeholder may be cautious and mention any piece of information she has, so that elicited quantity increases. On the other hand, the same stakeholder may be too cautious and share irrelevant information, thereby decreasing the quality of the information. In this paper, we therefore investigate the question of what changes whenever a stakeholder is committed or not to a RE project. We do so for the three main RECs, i.e., does commitment influence quality, quantity and/or efficiency of the elicitation documentation. Ultimately, we believe such contribution could help business analysts to select more accurately stakeholders so as to improve the chances of success for the system-to-be.

3) Contributions: Our contribution in this paper is twofold. Firstly, we discuss and clarify the distinction between the concept of involvement and commitment in elicitation, and we describe reasons why commitment may be an important criterion to account for when selecting stakeholders. Secondly, we survey 87 stakeholders in order to better understand how stakeholders' commitment to a RE project may influence the quality, quantity and efficiency of the elicitation documentation. Based on these results, we propose a commitment matrix, showing the relative advantage of involving stakeholders with various commitment levels. The matrix is shown in Figure 1 and reads as follows; if a business analyst needs to collect information that is, for example, clear, then she can involve any 


\begin{tabular}{|c|c|c|c|c|}
\hline & & \multicolumn{3}{|c|}{ Requirements Elicitation Challenges (RECs) } \\
\hline & & Quality & Quantity & Efficiency \\
\hline \multirow{4}{*}{ 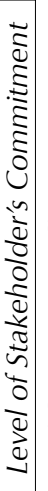 } & \multirow{4}{*}{ 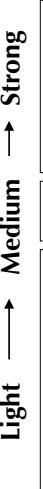 } & $\begin{array}{l}\text { Feasible: stakeholders share requests about the } \\
\text { future system that they believe are relevant, legal, } \\
\text { ethical, and not too extravagent given available } \\
\text { resources; }\end{array}$ & \multirow{2}{*}{$\begin{array}{l}\text { Granularity: stakeholders speak about details } \\
\text { related to the system or the way they expect the } \\
\text { latter to work; } \\
\text { Rules: stakeholders speak about laws, norms, } \\
\text { standards, their habits, their culture, or any } \\
\text { other constraint that shaped their behaviour. }\end{array}$} & $\begin{array}{l}\text { Scope: stakeholders discuss proactively about } \\
\text { topics, even though these topics have not been } \\
\text { suggested by the business analysts. }\end{array}$ \\
\hline & & $\begin{array}{l}\text { Certain: stakeholders only share information } \\
\text { about the system that they know for sure are true. }\end{array}$ & & \multirow{2}{*}{$\begin{array}{l}\text { Requirements: stakeholders discuss proactively } \\
\text { about their requirements from the future system, } \\
\text { even if not asked by the business analysts; } \\
\text { Domain: stakeholders discuss proactively about } \\
\text { the context of the system-to-be. }\end{array}$} \\
\hline & & & \begin{tabular}{|l|} 
Item: stakeholders speak about people, \\
objects, concepts or other systems related with \\
the system-to-be.
\end{tabular} & \\
\hline & & $\begin{array}{l}\text { Clear: stakeholders requests are easy to } \\
\text { understand; } \\
\text { Priority: stakeholders share requests with a clearly } \\
\text { defined order of priority. } \\
\text { Concise: stakeholders share requests that are not } \\
\text { too long, and do not take to much time to } \\
\text { communicate them. } \\
\text { Mandatory: stakeholders distinguish between } \\
\text { optional and mandatory requests }\end{array}$ & $\begin{array}{l}\text { Localization: stakeholders speak when and } \\
\text { where the system will be used; } \\
\text { Activity: stakeholders speak about the } \\
\text { intentions of the business; } \\
\text { Connection: stakeholders speak about } \\
\text { relationships or links between two ore more } \\
\text { agents of the business. }\end{array}$ & $\begin{array}{l}\text { Feedback: stakeholders provide feedback about } \\
\text { the way the project is going on, and what they } \\
\text { believe is good or bad; } \\
\text { Challenge: stakeholders never hesitate to } \\
\text { challenge or criticize a decision being made by } \\
\text { the business analysts. }\end{array}$ \\
\hline
\end{tabular}

Fig. 1: The Commitment Matrix

type of stakeholder, even those who are slightly commitment to the project, i.e., commitment is not important to account for when looking for clear information. However, if the analyst needs information that is certain, then she might have better chances to select strongly committed stakeholders. It is worthy to note that the survey is exploratory; our goal is to discover the impact of commitment on elicitation, and we have no apriori hypotheses to test in the study. Further validation of the matrix is therefore required before going on the formulation of actual recommendations for the selection of stakeholders during elicitation, based on commitment profiles.

4) Structure of the paper: The paper explains with more details how we obtained the commitment matrix illustrated in Figure 1, and is structured as follows. In Section 2, we review related work and distinguish between the concepts of commitment and involvement. We also provide a more accurate definition of commitment, and define the commitment hierarchy. In Section 3, we describe our survey design. We present our results in Section 4, discuss them in Section 5, and finally provide a conclusion to the present work in Section 6.

\section{RELATED WORKS AND DEFINITIONS}

Involving stakeholders as a way to improve the chances of development success is hardly new in RE and software engineering [11], [12]. It is often acknowledged that involvement is a good way to get faster to information about the domain, and to collect more accurately information about requirements [13], [7]. It is therefore frequently recommended as a best practice during elicitation [10]. Using a case study, [14] shows the different ways in which stakeholders (and more precisely customers) may be involved, and confirms the positive influence of involvement on the acquisition of information about the requirements. In [15], it is suggested that user involvement can be improved using a model-driven requirements approach. A systematic review of empirical studies about stakeholders involvement is proposed in [7], which confirms the topic has already been extensively discussed and tested in RE. Previous works seem to agree on a definition of involvement as follows:

The act for a stakeholder to communicate directly with the analysts, to share systematic feedback and to participate actively in the different stages of the development life-cycle [16].
Involvement requires that stakeholders are correctly identified and selected. This process is another important issue during elicitation [10]. In [17], authors argue that the selection of stakeholders somehow depends on their profile; depending of the type of information the analysts look for, stakeholders with different responsibilities, skills or knowledge will be targeted. In [18], the selection of stakeholders is discussed in the context of inter-organizational environments. Among other things, the author describes a matrix to classify stakeholders based on the interest they have in the system, and on the influence they might have on this project. In [19], a systematic review of stakeholders selection techniques is proposed. As already discussed, previous stakeholders selection techniques may lead to the identification of hundreds of stakeholders, and it is hardly feasible to involve all of them during elicitation. We suggest that commitment can be used as a criterion for involving only a subset of the pool of available stakeholders.

The concept of commitment has been the center of relatively little attention in RE. The distinction between commitment and involvement is sometimes made in RE literature [7], and stakeholders' commitment is sometimes acknowledged as a factor affecting the success of RE activities; in [20] for example, it is clearly stated that the geographical dispersion of teams likely decreases commitment of the stakeholders, which may be harmful to the RE project success. The concept is however scarcely studied as a main effect influencing quality, quantity or efficiency of elicitation documentation. To the best of our knowledge, no empirical research has gone on commitment during elicitation.

Given the relatively small attention that has been paid to commitment in RE, we clarify the concept of stakeholders commitment based on more mature definitions from psychology and management sciences. We use the influential definition of organizational commitment suggested in [21] as a baseline for our definition. We use that definition because it provides a set of criteria for commitment which are easily transferable to RE projects. Starting from Mowday's definition of organizational commitment, we define stakeholders commitment to a $\mathrm{RE}$ project as follows: the relative strength of a stakeholders identification with and involvement in an RE project. As in [21], it can be characterized by at least three factors:

- Acceptance - A strong belief in and acceptance of the RE 
TABLE I: Possible Commitment Profiles toward a RE Project

\begin{tabular}{|c|l|}
\hline Profile & Description \\
\hline Light & $\begin{array}{l}\text { Stakeholders who agree with the content, purpose and values of the } \\
\text { project, but unwilling to put much effort in the latter (Acceptance) }\end{array}$ \\
\hline Medium & $\begin{array}{l}\text { Stakeholders who agree with the content of the project, its purpose } \\
\text { and its values, and who are willing to help under some time and/or } \\
\text { resource constraints (Acceptance and Effort) }\end{array}$ \\
\hline Strong & $\begin{array}{l}\text { Stakeholders who agree with the content of the project, its purpose and } \\
\text { its values, and who are willing to help in the project with no limitations } \\
\text { on the time or resources (Acceptance, Effort and Membership) }\end{array}$ \\
\hline
\end{tabular}

project's goals and values;

- Effort - A willingness to exert considerable effort on behalf of the RE project;

- Membership - A strong desire to maintain membership in the RE project.

We see these three criteria as necessary, to some extent, in order to ensure a stakeholder is committed to a RE project. In case a stakeholder respects none of these criteria, she can be assumed to be uncommitted. If she respects one or more of theses criteria, the stakeholder is assumed to be somehow committed to the RE project. It is interesting to note that these three criteria build a hierarchy of commitment. For example, if there is Effort, then there is necessarily Acceptance, but not systematically Membership, i.e., there is a generalisation relationship between these different criteria. The commitment criteria can therefore be used to build a hierarchy of stakeholders' commitment. In other words, it is possible to find, in the pool of available stakeholders, stakeholders who have different levels of commitment. We summarize these different possible commitment profiles in Table $\mathrm{I}$

\section{EXPLORATORY STUDY - EMPIRICAL DESIGN}

Our objective is to investigate how commitment affects the significance of RECs, i.e., we want to show that commitment influences the quality, quantity and efficiency of the elicitation documentation. To explore this aspect, we use a survey. Based on the results of this survey, we have been able to draw the commitment matrix, as presented in our introduction. The survey we used was composed of three main sections.

\section{A. Procedure}

1) Assignment and Context: The first section was intended to introduce the subject to the context of the survey and the assignments. Subjects were told the survey was intended to understand how the implication of stakeholders may help in the design of a new information system. No more details were given about the goal of the study. Subjects were put into situation with the following paragraph:

We develop a new website for internal use in your company. We need to collect information about your expectations toward that website. Our goal is to understand what you expect from such website, and what you know about the future environment of that software.

The system was then described with more details. There were two different descriptions of the website, only one of which being submitted randomly to the subject: each time a subject opened the survey, a random number was computed that was used to select one scenario or the other, so that no subject faced the two descriptions. The objective was to provide stimuli in order to ensure the subject was clearly commitment or not toward the RE project that had been presented. Although the situation was purely hypothetical, we invited the subjects to recall the last project in which they had been involved, and use it as a global context to answer our survey. Ultimately, the decision to be committed or not was left to the stakeholder, i.e., we did not force subjects to be committed or not. The two possible descriptions were as follows:

- The website is a positive move: "Imagine that you have been waiting for the website for ages, that it will make your job much easier, will be fun and easy to use and will ease collaboration between colleagues";

- The website is a negative change: "Imagine that you are forced to used the new website, that it will change your routines and make interactions with colleagues more difficult, and that it need to be trained to use it".

2) Group Assignation: The second section was intended to measure the actual commitment of the stakeholder toward the RE project. Once subjects had read the description of the $\mathrm{RE}$ project that had been assigned to them, we measured how committed they were toward the latter, i.e., subjects were not forced into a group and were free to be committed or not to the RE project. We classified subjects in three different groups. These groups correspond to the commitment profiles defined in Table 1, namely the Light commitment group (L), the Medium commitment group (M) and the Strong commitment group (H). To allocate a subject to one of these groups, we used the three criteria of commitment presented in Section 2. For each criterion, subjects were given a binary scale equivalent to a "Yes/No" answer. The questions were as follows:

- Acceptance: Do you agree with the purpose of the project?

- Effort: Do you agree to spend time on the project?

- Membership: Do you want to participate on the long term to this project?

Note again that the design enables subjects to face a negative change and still be committed to the project, i.e., although we expect a positive change to lead to stronger commitment, it may happen that a stakeholder is commitment to a RE project implying a negative change, or vice-versa.

3) Collecting Survey Data: The third section was intended to collect data about what information stakeholders would share during the elicitation, i.e., our goal here was to actually measure RECs. The three main hypotheses we wanted to test - namely, that commitment influences quantity, quality and efficiency of the documentation - were however too coarse to be validated as such. As a consequence, we divided each REC in a series of more specific questions, easier to measure and hence to explore. We call these sub-questions variables in the rest of this paper. Each variable of the survey was measured using a five level Likert scale of agreement. Subjects were asked how they were feeling about a given sentence, and had to select one answer among Strongly Disagree, Disagree, Neither Agree nor Disagree, Agree or Strongly Agree. The set of variables that we used in our survey is reported in Table III 
The quality of documentation REC was studied using some of the quality criteria defined in the quality requirement framework suggested in [22]. Although the sentences shared by stakeholders during elicitation were not proper requirements, we consider the previous criteria can still be considered as ways to evaluate quality of information. The quantity of documentation REC was studied using some dimensions of context defined in [23]. Our objective here was simply to provide examples of topics that might be discussed during interviews, and see how subjects were behaving toward the latter. What we wanted to show is that, depending on their commitment to the RE project, stakeholders tend to discuss different topics to different extents. We do not claim such list is a way to ensure completeness of the elicitation; it simply comes as a basis to compare the groups. The efficiency of documentation REC is, to the best of our knowledge, a less common subject in RE, and we did not find any existing list of efficiency variables for RE. We therefore proposed some variables based on our experience. Given the exploratory nature of this study, we do not expect this decision to have a significant impact on our results.

\section{B. Subjects}

The subjects we targeted to answer previous survey are frequent users of information systems. Users represent an important proportion of the stakeholders' population, and are actors of the business whose commitment can significantly vary across a same RE project. Users therefore represents an interesting population for the present study. To make sure subjects understand the problem of sharing requirements about a system-to-be, we set three requirements on the demographic of our population: it was mandatory (i) to have at least a oneyear working experience in a company, (ii) to be more than 20 years old and (iii) to be frequent user of an information system for professional use. We used Amazon Mechanical

TABLE II: REC Variables in our Survey

\begin{tabular}{|c|c|}
\hline Quality & It is important to me to... \\
\hline Clear & ...explain my expectations with clear and precise words \\
\hline Prioritized & ...tell you which of my expectations are more/less important \\
\hline Concise & ...explain my expectations concisely and quickly \\
\hline Feasible & ...make sure what I say is relevant, legal, ethical, not extravagant \\
\hline Certain & ...share information I am certain of, avoid things I am not sure of \\
\hline Mandatory & $\begin{array}{l}\text {...make sure, during the interview, that you have understood the most } \\
\text { important expectations I have from the system }\end{array}$ \\
\hline Quantity & It is important to me to... \\
\hline Items & $\begin{array}{l}\text {... who will use the software, devices on which the software will run, } \\
\text { documents which need to be used and produced by the software }\end{array}$ \\
\hline Localization & $\begin{array}{l}\text {...how frequently the software will be used and the location where } \\
\text { the software will be used }\end{array}$ \\
\hline Rules & $\begin{array}{l}\text {...rules which apply to the software in my company, and laws and } \\
\text { norms that the software should comply with }\end{array}$ \\
\hline Connections & $\begin{array}{l}\text {...the relationships between the people in my company, in order to } \\
\text { understand my expectations from the software }\end{array}$ \\
\hline Activities & $\begin{array}{l}\text {...why my company needs the software, what the purpose of that } \\
\text { software is, which problems it should help solve }\end{array}$ \\
\hline Granularities & $\begin{array}{l}\text {...the programming language in which the software should be made, } \\
\text { the specifics of the databases it will manage, the components of the } \\
\text { software, the metrics used to evaluate the quality of that software }\end{array}$ \\
\hline Efficiency & It is important to me to... \\
\hline Requirement & ...share pro-actively information about what I expect \\
\hline Domain & $\begin{array}{l}\text {...share pro-actively the information I have about the environment in } \\
\text { which the software will operate }\end{array}$ \\
\hline Feedback & ...give feedback about the way the project is going on \\
\hline Challenge & $\begin{array}{l}\text {...ask questions about choices made by the designers, or challenges } \\
\text { decisions I do not agree with }\end{array}$ \\
\hline Scope & ...discuss topics other than those suggested by the business analysts \\
\hline
\end{tabular}

Turk (simply MTurk hereafter) to collect data for our study. Mturk enables to access a large panel of subjects, who are sufficiently diverse to be representative of actual stakeholders' population. MTurk is based on a reward system: participants have to select some tasks that they accept to complete in exchange for a certain amount of money, determined a priori by the experimenter. We discuss the validity issues related to this approach in next section. We collected the answers of 87 subjects, all living in USA at the moment of the study: $49 \%$ of them were women, $90 \%$ were between 26 and 54 years old, and $85 \%$ had at least under-graduated. The most represented business fields were services to people $(16 \%)$, retail trade $(14 \%)$ and information technologies $(11.5 \%)$.

\section{Methodological Notes}

A pre-study was performed; we submitted our survey to a dozen of stakeholders, and asked them to provide feedback about the overall readability, fluency and clarity of the survey. Based on those feedbacks, we significantly improved the survey. Answers collected during this preliminary data collection have not been included in our final data-set. The design we used in this research is a survey. As any empirical design, it may be subject to some internal and external threats. Threats to internal validity include the selection bias, the experimental arrangement and the confounding bias. To deal with them, we left group assignation as a responsibility of the subjects (based on commitment criteria), we randomized the position of questions within our survey, so that the combination of questions was always different for different subjects, and we paid attention to use valid scales to measure our variables. The main threat to external validity is the use of MTurk (see next section); we had small control on the people answering the survey, and people may not be have been sufficiently involved in answering correctly the survey. To reduce this risk, we only collected answers from Master MTurk profiles, i.e., elite groups of MTurkers who have demonstrated accuracy on specific types of HITs (i.e. survey) on the Mechanical Turk marketplace. We also made use of "attention check questions". These are questions where subjects are asked to remember some simple words and to encode the latter at the end of the survey; they enable to test attention of subjects and detect spammers. Subjects who did not answer correctly to those questions were excluded from our results.

\section{ANALysis OF Results}

This section discusses the results that we obtained using our survey 11 We perform comparison between several groups and want to see if there are significant differences between these groups. ANOVA tests can be used to achieve such conclusion. Given that our data are ordinal, we resort to the non-parametric equivalent of the ANOVA for multiple groups, namely the Kruskal-Wallis test. The null hypothesis being tested is then that none of the groups being tested statistically dominate any other one, i.e, if we can reject this hypothesis, we can conclude that there are statistical differences between at least two of the three commitment profiles. To build the commitment matrix, we put variables for which the null hypothesis cannot be rejected at the bottom of the matrix; these are the variables for which we have no indications that commitment influences their

\footnotetext{
${ }^{1}$ Data are accessible at http://perso.unamur.be/ cburnay/Commitment/
} 
value, so that they can be elicited from any type of commitment profile. Then, we look at the variables for which we can reject the null hypothesis. For these variables, we have indications that commitment influences their value, and we can then report them in the relevant layer of our matrix. Since the tests we run do not indicate the direction of the influence, we also resort to graphical representations of the survey data to complement our analysis. An important concern when measuring RECs Quantity, Quality and Efficiency of the documentation - with several variables is the internal validity of these variables; for example, do the Clear, Concise, Certain, ... variables reliably measure the same latent Quality variable. The Cronbach's alpha is one common measure of such internal consistency. It is typically used in surveys where several Likert questions are used to build one main scale - such as in our survey - and the reliability of that scale has to be measured. It is common to interpret the Cronbach's Alpha with the following limits: a value from 0 to .50 is usually small and suggests low internal validity of the scale, a value between .50 and .70 is low but acceptable, while values above .70 are high and suggest that the scale has high internal validity. We computed an alpha of 0.7139 for the Quality scale, an alpha of 0.7322 for the Quantity scale, and an alpha of 0.8585 for the Efficiency scale. We therefore conclude our scales provide reliable ways to observe RECs.

1) Quality Variables: Results for the Quality REC are presented in top area of Table III The null hypothesis can be rejected if the p-value is smaller than some significance levels, which in this study are: $1 \%=* * *, 5 \%=* *$ and $10 \%=*$. We observe that answers from the three commitment groups only significantly differ for the Feasible and Certain variables. The tests do not enable to conclude more about the other variables that we used to measure quality. Columns $\mathrm{a}$ and $\mathrm{b}$ in Table IV show the distribution of answers across the groups ( $\mathrm{L}=\mathrm{Low}$, $\mathrm{M}=$ Medium and $\mathrm{H}=$ High profile), for these two significant variables. We observe that a larger part of the strong and medium commitment profiles strongly agrees with the fact that they would pay attention to share feasible and certain information. While the difference is smaller on the agree answer, we also observe that the light commitment profile tends to disagree more frequently than the two other groups for such statements. This brings us to the conclusion that, in order to increase the chances of collecting Feasible and Certain information, business analysts should involve stakeholders with a medium commitment profile or higher.

2) Quantity Variables: Results for the Quantity REC are presented in the middle area of Table [III] We observe that the three commitment groups significantly differ for the Items, Rules and Granularities variables. We cannot conclude anything about the other quality variables. Columns $\mathrm{c}, \mathrm{d}$ and $\mathrm{e}$ in Table IV show the distribution of answers for the three significant variables. We observe no clear differences between the medium and strong commitment profiles, for the Items variable. This suggests that business analysts can involve any of these two profiles to reduce the risk of omissions about objects and agents who (will) interact with the system. On the contrary, we observe that strong commitment profile strongly agrees more frequently than the two other profiles when considering rules and granularities. Besides, the light and medium profiles answered more frequently that they (strongly) disagree with sharing such information. This brings us to the conclusion
TABLE III: Kruskall-Wallis Tests on the Survey Variables

\begin{tabular}{|l|c|c|c|}
\hline Quality Variables (Freedom degree $=2$ ) & X-Squared & P-Value & Significance \\
\hline Clear & 1.6192 & 0.445 & - \\
\hline Priority & 2.2625 & 0.322 & - \\
\hline Concise & 1.9774 & 0.372 & - \\
\hline Feasible & 7.5268 & 0.023 & $* *$ \\
\hline Certain & 5.9537 & 0.051 & $*$ \\
\hline Mandatory & 0.4599 & 0.7946 & - \\
\hline \hline Items & 4.6929 & 0.096 & $*$ \\
\hline Localization & 3.9085 & 0.142 & - \\
\hline Rules & 4.8234 & 0.089 & $*$ \\
\hline Connections & 0.0413 & 0.9796 & - \\
\hline Granularities & 7.6043 & 0.022 & $* *$ \\
\hline Activities & 4.3940 & 0.111 & - \\
\hline \hline Requirements & 13.4056 & 0.001 & $* * *$ \\
\hline Domain & 10.6544 & 0.004 & $* * *$ \\
\hline Feedback & 3.1640 & 0.205 & - \\
\hline Challenge & 2.7031 & 0.2588 & - \\
\hline Scope & 9.6774 & 0.008 & $* * *$ \\
\hline
\end{tabular}

that analysts should involve strongly committed stakeholders if they want to reduce the risk of missing information about the constraints applying on the system or about the details related to how the system will operate.

3) Efficiency Variables: Results for the Efficiency REC are presented in the bottom area of Table III The three commitment groups significantly differ for the Requirements, Domain and Scope variables. We cannot conclude more about the other efficiency variables. Columns $\mathrm{f}, \mathrm{g}$ and $\mathrm{h}$ in Table IV show the distribution of answers across the groups, for these three significant variables. Although differences are small, we observe that the strong commitment profile seems to be more likely to (strongly) agree when being asked if they would be pro-active in sharing their requirements. This brings us to the recommendation that business analysts should involve strongly committed stakeholders if they wish to collect more efficiently information about requirements. Both medium and strong commitment groups (strongly) agree that they would share spontaneously information about the domain or the scope of the project, while the light commitment group (strongly) disagrees more frequently with these same variables. This suggests business analysts should involve stakeholders that are moderately committed (or higher) to the project, so as to increase the chances of getting efficiently information about the environment of the system, or details about its scope.

\section{DISCUSSION}

Our results clearly show that commitment is one factor that seems to have a potentially significant impact on the overall success of the elicitation process. However, it is interesting to note that the effect of commitment is only partial; it seems that commitment does not influence all the aspects of the elicitation challenges we identified, but only some specific concerns. The question is then to understand why a variable is impacted or not by commitment. Under such perspective, our study becomes a tool for identifying more specific research questions to be investigated in some future research. A possible interesting direction is to study the relation between the complexity of an elicitation task and the overall commitment level. For example, simple tasks like sharing clear information, speaking about connections or challenging people seem less likely to fail even when commitment profile is low. However, when the tasks require more effort - that is, when tasks are more complex -, 
TABLE IV: Distribution of Quality, Quantity and Efficiency Answers by Commitment Profiles

\begin{tabular}{|c|c|c|c|c|c|c|c|c|c|c|c|c|c|c|c|c|c|c|c|c|c|c|c|c|}
\hline & \multicolumn{3}{|c|}{ Certain (a) } & \multicolumn{3}{|c|}{ Feasible (b) } & \multicolumn{3}{|c|}{ Item $(\mathrm{c})$} & \multicolumn{3}{|c|}{ Granularity (d) } & \multicolumn{3}{|c|}{ Rules (e) } & \multicolumn{3}{|c|}{ Requirements (f) } & \multicolumn{3}{|c|}{ Domain $(\mathrm{g})$} & \multicolumn{3}{|c|}{ Scope (h) } \\
\hline & $L$ & $M$ & $H$ & $L$ & $M$ & $H$ & $L$ & $M$ & $H$ & $L$ & $M$ & $H$ & $L$ & $M$ & $H$ & $L$ & $M$ & $H$ & $L$ & $M$ & $H$ & $L$ & $M$ & $H$ \\
\hline Str. Disagree & 0 & 0 & 0 & 0 & 0 & 0 & 0 & 0 & 0 & 5 & 6 & 2 & 5 & 0 & 2 & 0 & 0 & 0 & 0 & 0 & 0 & 0 & 0 & 0 \\
\hline Disagree & 5 & 0 & 2 & 10 & 6 & 2 & 14 & 18 & 2 & 33 & 41 & 22 & 19 & 29 & 10 & 29 & 0 & 2 & 29 & 6 & 0 & 38 & 24 & 4 \\
\hline Neutral & 19 & 6 & 4 & 29 & 0 & 10 & 19 & 6 & 8 & 24 & 12 & 8 & 19 & 0 & 12 & 33 & 18 & 4 & 19 & 6 & 8 & 24 & 0 & 20 \\
\hline Disagree & 57 & 65 & 51 & 46 & 65 & 51 & 52 & 47 & 61 & 29 & 41 & 45 & 52 & 65 & 53 & 19 & 65 & 67 & 38 & 59 & 63 & 29 & 65 & 53 \\
\hline Str. Agree & 19 & 29 & 43 & 14 & 29 & 37 & 14 & 29 & 29 & 10 & 0 & 22 & 5 & 6 & 22 & 19 & 18 & 27 & 14 & 29 & 29 & 10 & 12 & 22 \\
\hline
\end{tabular}

commitment appears to be a more important variable. Another interesting direction is to study the link between commitment and the recency of a task. In fact, it seems that more recurring cognitive tasks such as sharing prioritized information, speaking about localizations or giving feedback are less likely to fail even if the commitment is low. In practice, these tasks are likely to occur more frequently and are likely to be more commonsense to stakeholders, so that even low commitment profiles can deal with them correctly.Overall, we believe that investigating such questions might help in the formulation of additional guidelines for the selection and involvement of stakeholders.

\section{CONCLUSIONS}

In this paper, we discuss the concept of stakeholders' commitment to a RE project, why it can be a relevant criterion to select, among the large pool of stakeholders, those that should be involved in the elicitation, and how it differs from involvement. Based on the survey of 87 stakeholders, we propose the commitment matrix, which describes the relative advantages of involving stakeholders with light, medium and strong commitment profiles. We observe that stakeholders with different commitment share information which nature may vary, in terms of quality, quantity or efficiency. It suggests that, depending on the type of information a business analyst is looking for, the commitment level of a stakeholder is more or less important to account for. While the paper does not provide any elicitation methodology, it suggests ways for accounting for commitment in order to better deal with the RECs. The commitment matrix is an exploratory study, not a proper empirical validation; readers should bear in mind that it builds on a small sample, and that further validation is required before formulating recommendations for the selection of stakeholders. We believe this does not hold us back from drawing relevant conclusions about how commitment affects RECs, and about what research is necessary in the future so as to better deal with such aspect during elicitation.

\section{REFERENCES}

[1] M. G. Christel and K. C. Kang, "Issues in requirements elicitation," Technical Report CMU/SEI-92-TR-12 ESC-TR-92-012, 1992.

[2] D. Zowghi and C. Coulin, "Requirements Elicitation : A Survey of Techniques , Approaches, and Tools," in Engineering and managing software requirements, C. Aurum, Aybüke and Wohlin, Ed. Springer Berlin Heidelberg, 2005, pp. 19-46.

[3] A. Sutcliffe and P. Sawyer, "Requirements elicitation: Towards the unknown unknowns," in Proc. 21st IEEE International Requirements Engineering Conference (RE). IEEE, Jul. 2013, pp. 92-104.

[4] A. M. Davis, O. Dieste, A. M. Hickey, N. Juristo, A. Moreno, and M., "Effectiveness of requirements elicitation techniques: Empirical results derived from a systematic review," in Proc. 14th IEEE International Conference on Requirements Engineering, 2006, pp. 179-188.

[5] A. M. Hickey and A. M. Davis, "A unified model of requirements elicitation," Journal of Management Information Systems, vol. 20, no. 4, pp. 65-84, 2004.
[6] R. Palanisamy and J. L. Sushil, "User Involvement in Information Systems Planning Leads to Strategic Success: An Empirical Study," Journal of Services Research, vol. 1, no. 2, pp. 125-157, 2001.

[7] M. Bano and D. Zowghi, "Users' involvement in requirements engineering and system success," Proc. 3rd International Workshop on Empirical Requirements Engineering (EmpiRE), pp. 24-31, Jul. 2013.

[8] A. Pouloudi, "Stakeholder analysis as a front-end to knowledge elicitation," AI \& Society, vol. 11, no. 1-2, pp. 122-137, Mar. 1997.

[9] R. K. Mitchell, B. R. Agle, and D. J. Wood, "Toward a Theory of Stakeholder Identification and Salience: Defining the Principle of Who and What Really Counts," The Academy of Management Review, vol. 22, no. 4, pp. 853-886, 1997.

[10] M. Glinz and R. J. Wieringa, "Stakeholders in Requirements Engineering," IEEE Software, vol. 24, no. 2, pp. 18-20, 2007.

[11] D. Robey and D. Farrow, "User Involvement in Information System Development: A Conflict Model and Empirical Test," Management Science, vol. 28, no. 1, pp. 73-85, 1982.

[12] H. Barki and J. Hartwick, "Rethinking the Concept of User Involvement," MIS Quartly, vol. 13, no. 1, pp. 53-63, 1989.

[13] S. Kujala, M. Kauppinen, L. Lehtola, and T. Kojo, "The role of user involvement in requirements quality and project success," in Proc. 13th IEEE International Requirements Engineering Conference, 2005, pp. 75-84.

[14] J. Kabbedijk, S. Brinkkemper, S. Jansen, and B. van der Veldt, "Customer Involvement in Requirements Management: Lessons from Mass Market Software Development," in Proc. 17th IEEE International Requirements Engineering Conference. Ieee, 2009, pp. 281-286.

[15] J. M. Rivero, E. R. Luna, J. Grigera, and G. Rossi, "Improving user involvement through a model-driven requirements approach," Proc. $3 r d$ International Workshop on Model-Driven Requirements Engineering (MoDRE), pp. 20-29, Jul. 2013.

[16] L. Damodaran, "User involvement in the systems design process - a practical guide for users," Behaviour \& Information Technology, vol. 15, no. 6, pp. 363-377, 1996.

[17] J. Coughlan, M. Lycett, and R. D. Macredie, "Communication issues in requirements elicitation: a content analysis of stakeholder experiences," Information and Software Technology, vol. 45, no. 8, pp. 525-537, 2003.

[18] L. C. Ballejos and J. M. Montagna, "Method for stakeholder identification in interorganizational environments," Requirements Engineering, vol. 13, no. 4, pp. 281-297, Sep. 2008.

[19] C. Pacheco and I. Garcia, "A systematic literature review of stakeholder identification methods in requirements elicitation," Journal of Systems and Software, vol. 85, no. 9, pp. 2171-2181, Sep. 2012.

[20] D. Damian and D. Zowghi, "The impact of stakeholders' geographical distribution on managing requirements in a multi-site organization," in Proc. IEEE Joint International Conference on Requirements Engineering, 2005, pp. 99-108.

[21] R. T. Mowday, R. M. Steers, and L. W. Porter, "The measurement of organizational commitment," Journal of Vocational Behavior, vol. 14, no. 2, pp. 224-247, Apr. 1979.

[22] A. Katasonov and M. Sakkinen, "Requirements quality control: a unifying framework," Requirements Engineering, vol. 11, no. 1, pp. 42-57, Oct. 2005.

[23] C. Burnay, I. J. Jureta, and S. Faulkner, "What stakeholders will or will not say: A theoretical and empirical study of topic importance in Requirements Engineering elicitation interviews," Information Systems, vol. 46, pp. 61-81, Nov. 2014. 